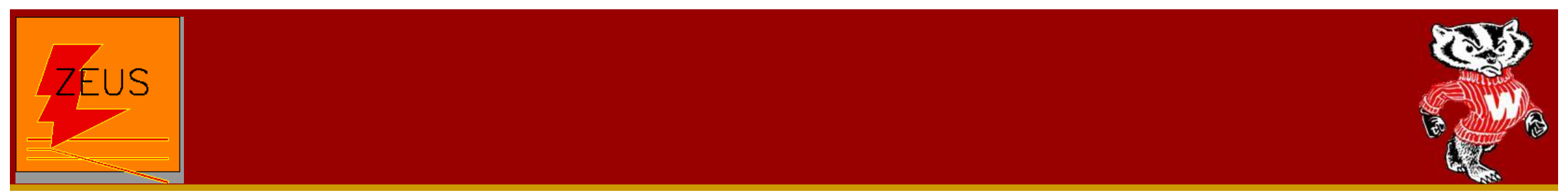

\title{
Photoproduction of Events with Rapidity Gaps Between Jets at ZEUS
}

\author{
Patrick Ryan \\ University of Wisconsin
}

On Behalf of the ZEUS Collaboration

\author{
DIS 2006 \\ Tsukuba, Japan \\ April 22, 2006
}




\section{Hard Diffractive Photoproduction}

Rapidity Gap Between Hadron

\& Proton Remnant

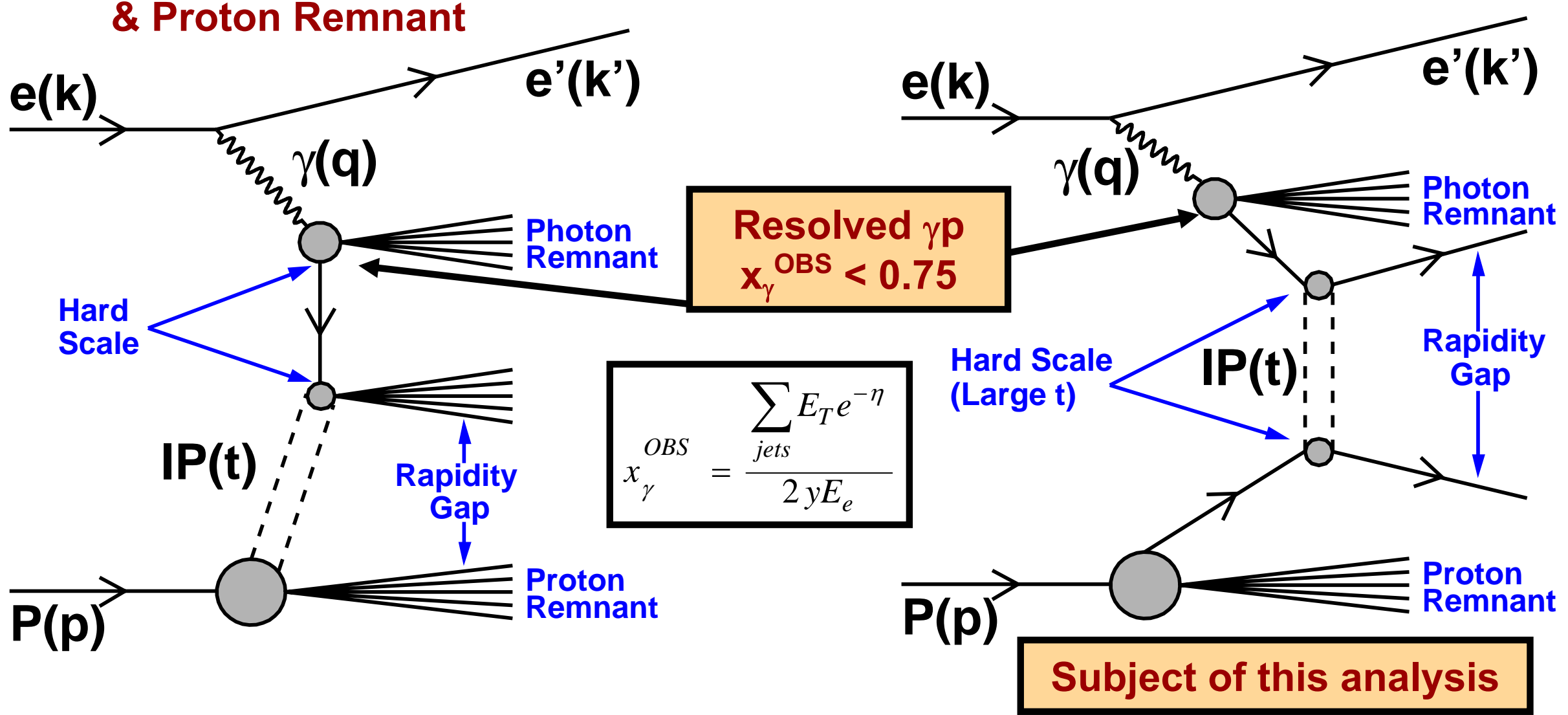

- Study the nature of the Pomeron

- Observe Color-Singlet exchange

- Hard Scale allows application of PQCD to diffractive process 


\section{Rapidity Gaps between Jets}

Color-Singlet Exchange

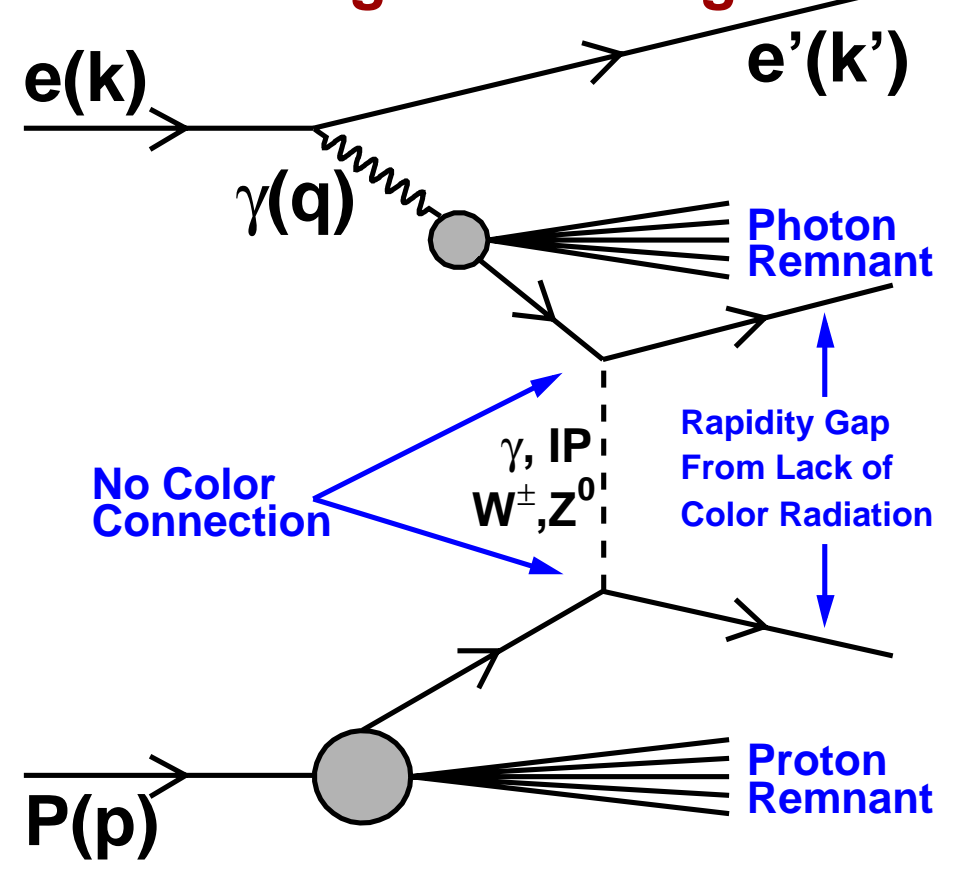

Color-Non-Singlet Exchange

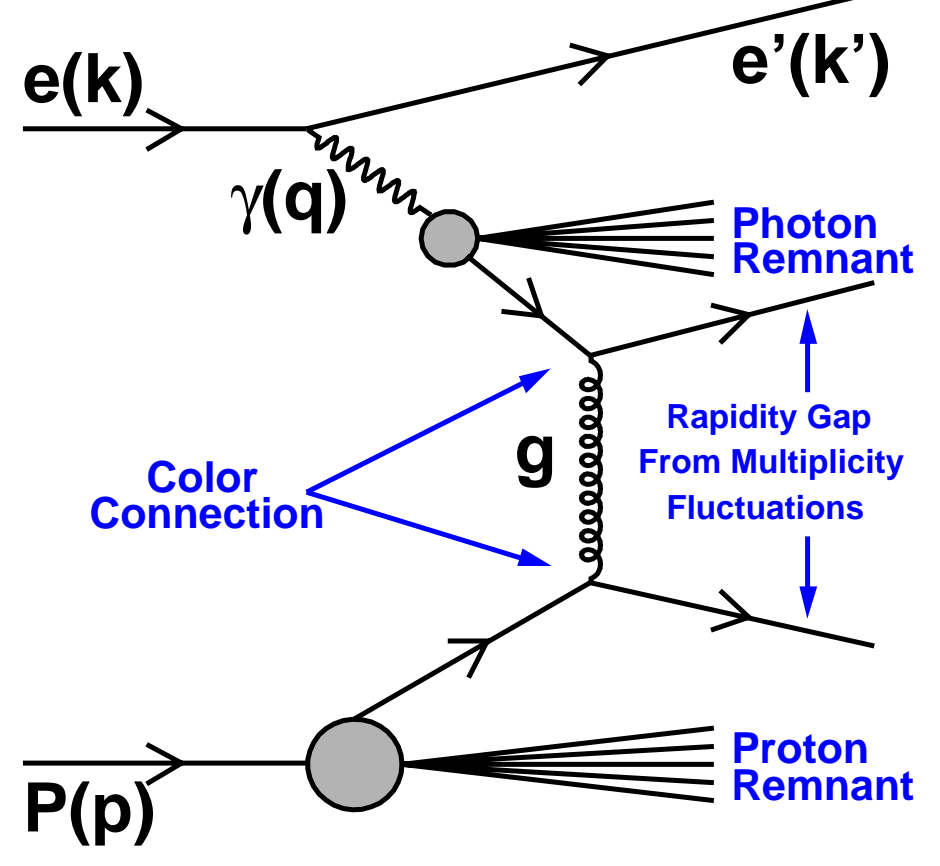

- 2 Sources of Rapidity Gaps between Jets

- Color-singlet Exchange

- Lack of color radiation produces gap

- Example: Pomeron

- Color-Non-Singet Exchange

- Fluctuations in particle multiplicity produces gap

- Non-diffractive 


\section{Rapidity Gap Topology}

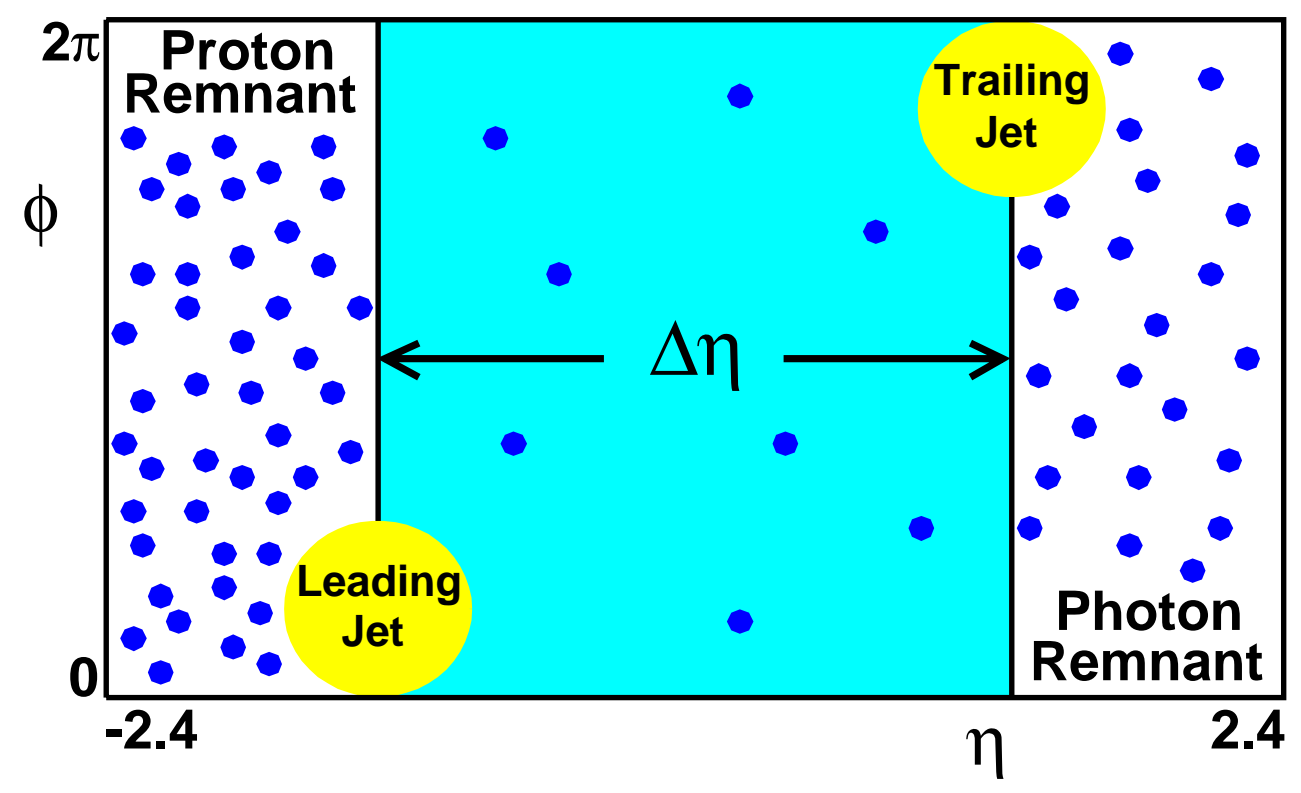

- Distance between leading and trailing jet centers: $\Delta \eta$

- $E_{T}$ Gap: Total $E_{T}$ of jets between leading and trailing jet centers

- Gap Event has small energy in Gap: $E_{T}{ }^{G a p}<E_{T}$ Cut

- Gap definition based on $E_{T}$ better than that based on multiplicity

- Collinear and infrared safe

- Gap spans between centers of leading \& trailing jets (increased statistics) 


\section{The Gap Fraction $f(\Delta \eta)$}

Dijet Events with large Rapidity separation between jets \& $\mathrm{E}_{\mathrm{T}} \mathrm{Gap}<\mathrm{E}_{\mathrm{T}}$ Cut

$$
f(\Delta \eta)=\frac{d \sigma_{g a p} / d \Delta \eta}{d \sigma / d \Delta \eta}
$$

All Dijet Events with large Rapidity separation between jets

$\sigma_{\text {gap }}=\sigma_{\text {gap }}^{\text {singlet }}+\sigma_{\text {gap }}^{\text {non-singlet }}$

- Color Singlet

- Gap created by lack of color flow

- $\mathbf{f}(\Delta \eta)$ constant in $\Delta \eta$

- Color Non-Singlet

- Gap created by multiplicity fluctuations

Expectation for Behavior of Gap Fraction (J. D. Bjorken, V. Del Duca, W.-K. Tung)

- $f(\Delta \eta)$ decreases exponentially with $\Delta \eta$ 


\section{ZEUS Comparison to Previous ZEUS and H1 Measurements}

\section{ZEUS 1995}

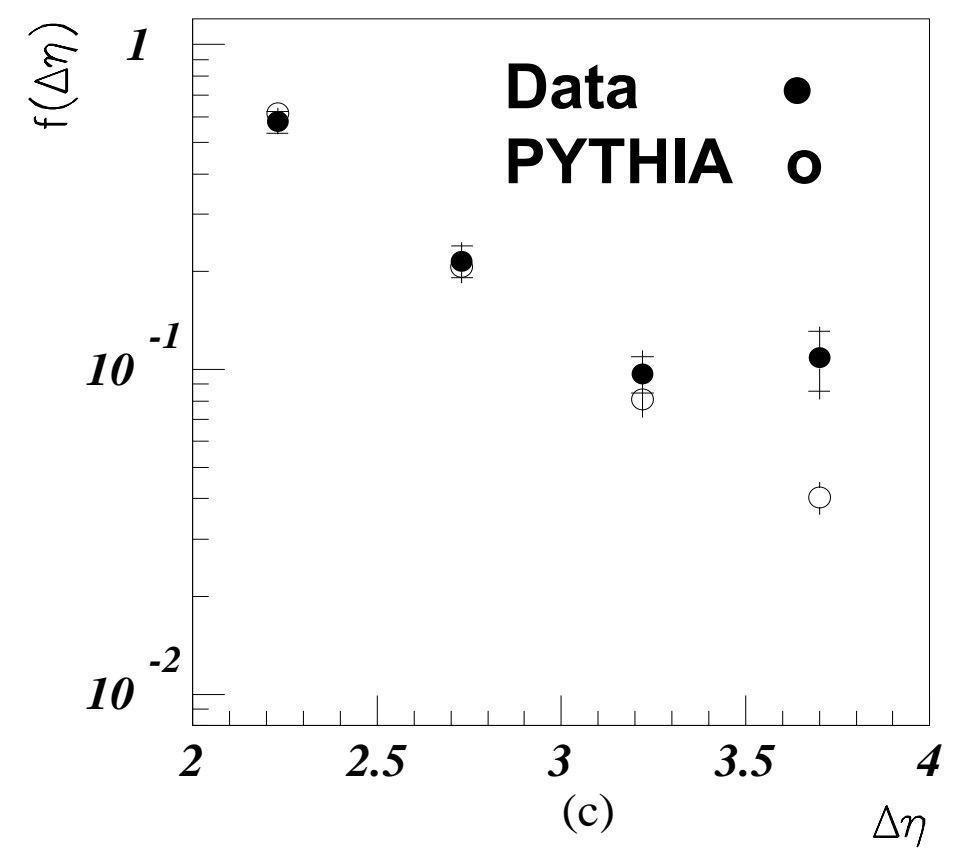

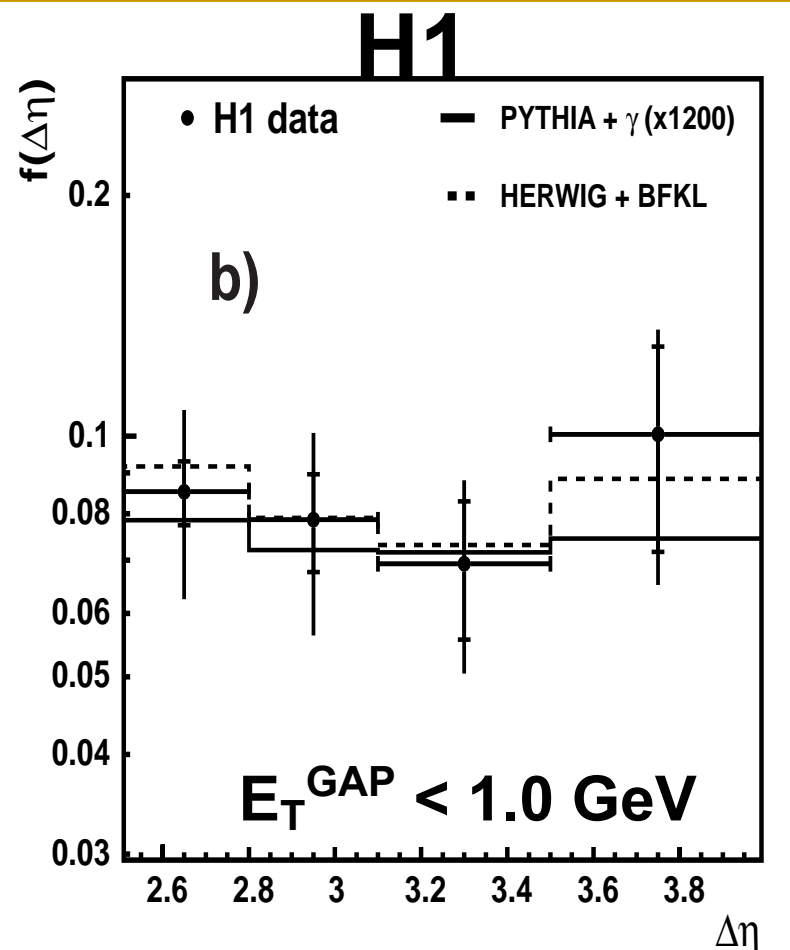

- ZEUS 1995: Gap Fraction defined by multiplicity

- Data above Non-Color-Singlet PYTHIA

- H1 2002: Gap Fraction for $E_{T}$ Gap $<1.0 \mathrm{GeV}$

- Data above NCS MC but Data described by NCS+CS MC

- $6.6 \mathrm{pb}^{-1}$ of Lumi 


\section{ZÉus Simulation of Color-Non-Singlet and
Color-Singlet MC}

- PYTHIA 6.1 \& HERWIG 6.1

- Used to simulate Color-Non-Singlet and Color-Singlet Events

- Shown to describe $\gamma p$ data

- Use different hadronization models

- Direct, Resolved, Color-Singlet MC generated separately

- Color-Non-Singlet MC

- Resolved MC includes Multi-Parton Interactions (MPIs)

- Color-Singlet (CS) Exchange MC

- HERWIG: BFKL Pomeron as exchange object

- Includes MPIs

- PYTHIA: High-t $\gamma$ exchange

- Used to match data only - Rapidity Gap not due to photon exchage

- Does not include MPIs 


\section{Monte Carlo Tuning}

- Modified Default ZEUS MC parameters

- Tuning based on JetWeb parameters (Global fit to collider data)

- Tuned $p_{T}{ }^{\text {Min }}$ to ZEUS $E_{T}{ }^{\text {GAP }}$ distributions (shown later)

- Tuned PYTHIA 6.1

- Proton PDF: CTEQ 5L (Set 46)

- Photon PDF: SaS-G 2D

$p_{T}{ }^{\text {Min } 1}: p_{T}$ of hardest interaction

$p_{T}{ }^{\text {Min } 2:} p_{T}$ of all secondary interactions

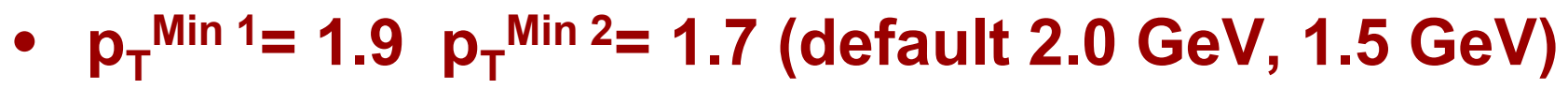

- Tuned HERWIG 6.1

- Proton PDF: CTEQ 5L (Set 46)

- Photon PDF SaS-G 2D

- Square of factor to reduce proton radius: 3.0 (default 1.0)

- Probability of Soft Underlying Event: 0.03 (default 1.0)

- $P_{\mathrm{T}}{ }^{\mathrm{MIN} 1}=2.7 \mathrm{GeV}$ (default $1.8 \mathrm{GeV}$ ) 


\section{Data Acceptance Corrections}

- Correct data for acceptance: Detector $\rightarrow$ Hadron level

- Step 1: Dir and Res relative amounts fit to Data: $x_{\gamma}{ }^{\text {OBS }}$ distribution

- PYTHIA - Detector Level

- 28\% Direct

- $72 \%$ Resolved

- HERWIG - Detector Level

- 44\% Direct

- 56\% Resolved

- Step 2: Non-Color Singlet \& Color Singlet relative amounts fit to Data: $E_{\text {TOT }}$ for $E_{T}{ }_{T}$ AP $<1.5 \mathrm{GeV}$ 


\section{Rapidity Gap Event Selection}

ZEUS 1996-97 Data (38 $\left.\mathrm{pb}^{-1}\right)$

Trigger Selection:

FLT, SLT, and TLT requirements to select dijet photoproduction events

Clean Photoproduction Sample:

Reject events having Electron with $\mathrm{E}_{\mathrm{e}}>5 \mathrm{GeV}$ AND $\mathrm{y}_{\mathrm{e}}<0.85$

$$
\begin{aligned}
& \Sigma \mathrm{p}_{\mathrm{T}} / \Sigma \sqrt{ } \mathrm{E}_{\mathrm{T}}<2 \mathrm{GeV}^{1 / 2} \\
& \left|z_{v t x}\right|<40 \mathrm{~cm} \\
& 0.2<y_{\mathrm{JB}}<0.85 \\
& \mathrm{E}_{\mathrm{T}}{ }^{1,2}>6.0,5.0 \mathrm{GeV} \\
& \left|\eta^{1,2}\right|<2.4 \\
& 1 / 2\left|\eta^{1}+\eta^{2}\right|<0.75 \\
& 2.5<\left|\eta^{1}-\eta^{2}\right|<4.0 \text { (Gap Definition) } \\
& \mathrm{E}_{\mathrm{T}} \mathrm{CUT}=0.5,1.0,1.5,2.0 \mathrm{GeV}
\end{aligned}
$$

$\sim 70,000$ Events in Inclusive Sample 


\section{ZEUS Acceptance Corrected Data vs MC
$\mathrm{E}_{\mathrm{T}}$ Gap Cross Section}

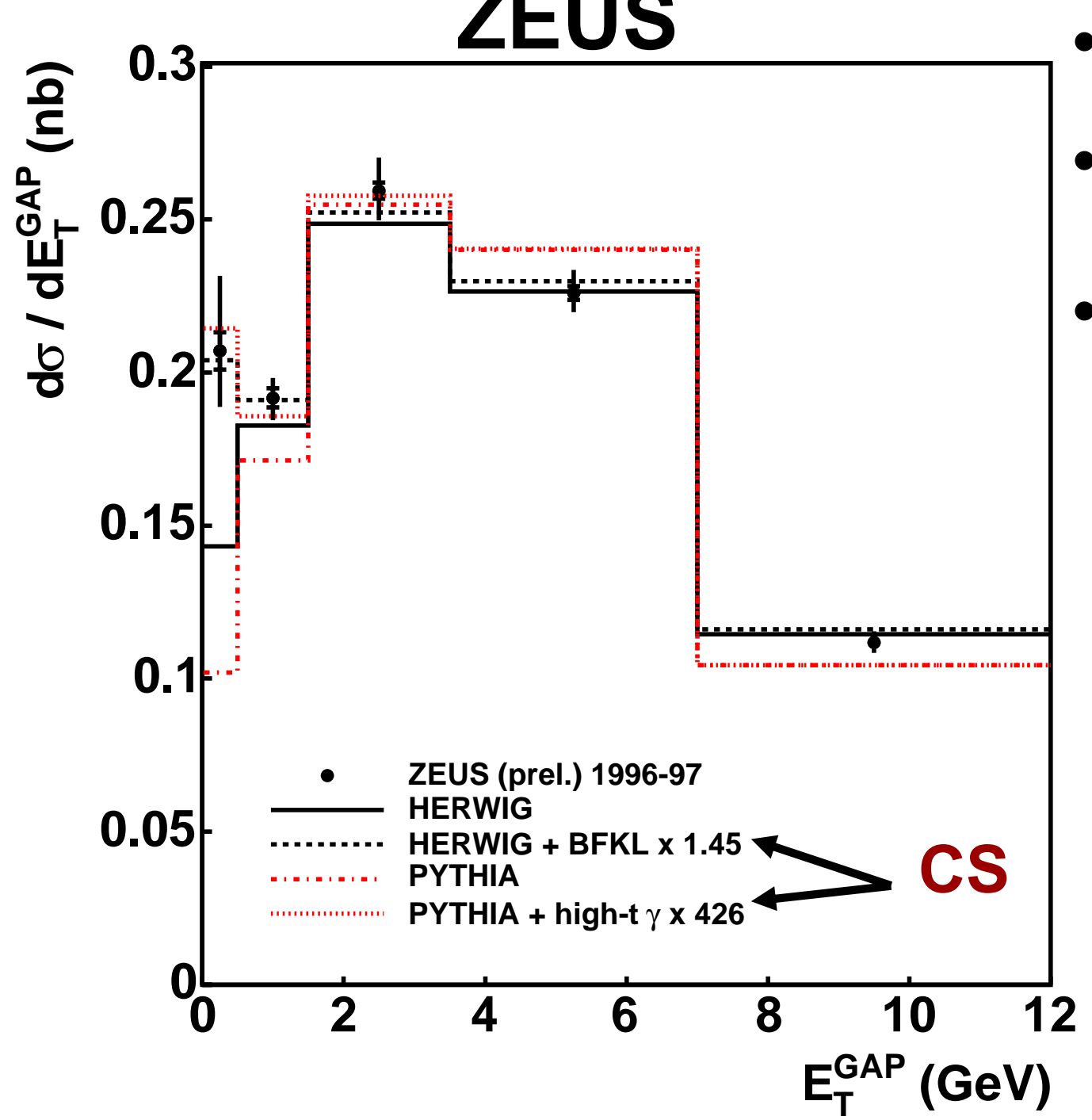

- Acceptance Correction

- Average of PYT \& HER

- Systematic Errors from HER

- Difference between HER \& PYT values added to systematic

- MCs fit to Data

- $\chi^{2}$ Minimization

- Yield Scale Factors

- HER: $1.01^{\star} \mathrm{NCS}+1.45^{\star} \mathrm{CS}$

- PYT: $1.25^{\star} \mathrm{NCS}+426 * \mathrm{CS}$

- High CS Scale Factor in PYTHIA due to High-t $\gamma$ exchange

Minimization of $\chi^{2}$ in fit to Data results in $\sim 3 \%$ CS contribution for both PYTHIA \& HERWIG 


\section{ZEus Acceptance Corrected Data vs MC
$\Delta \eta$ Cross Sections}

ZEUS

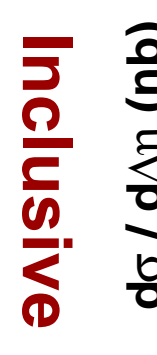

$\frac{0}{5}$
$\frac{1}{0}$
$\frac{0}{0}$
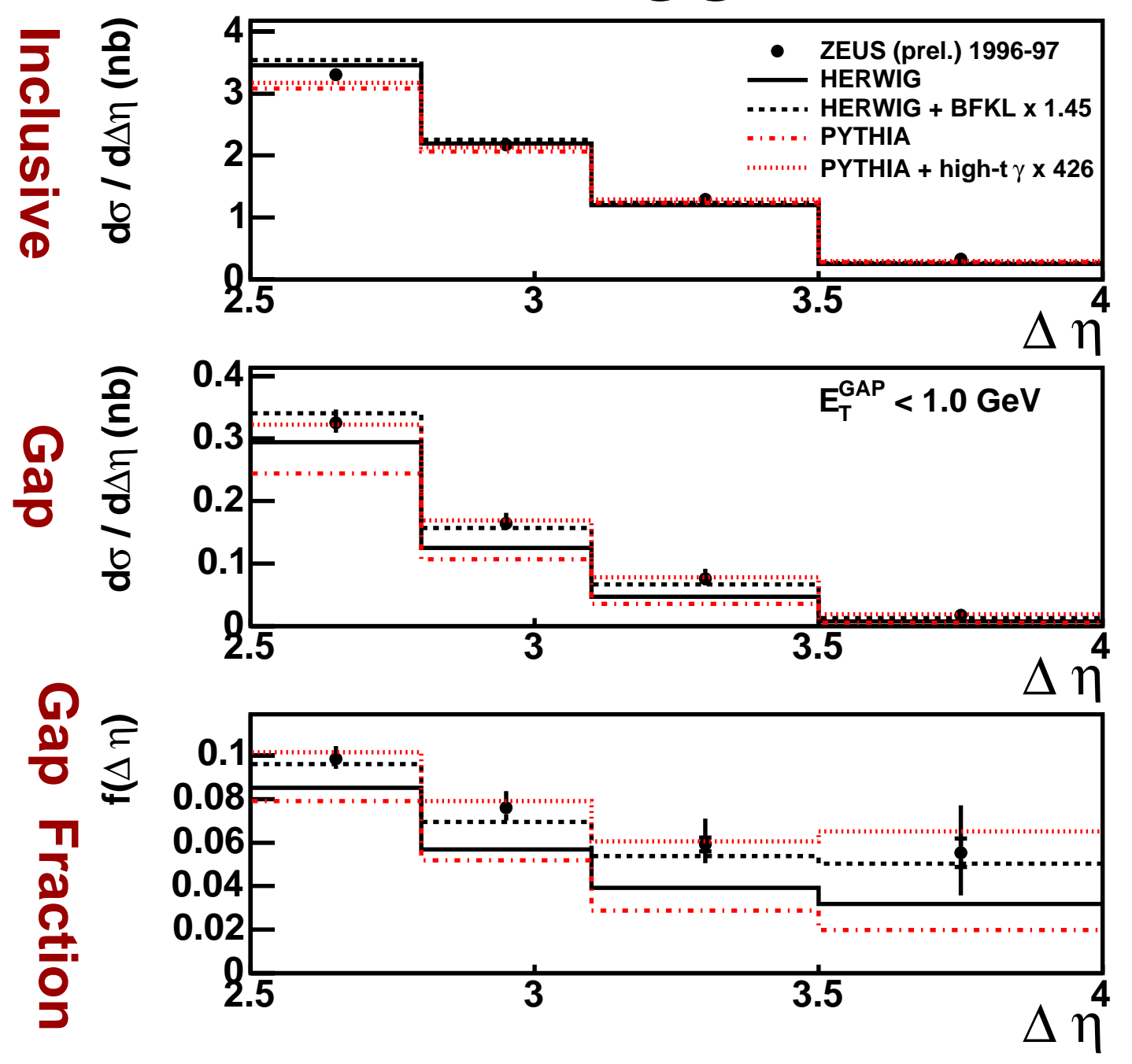

- MC with CS added describes data 


\section{ZEUs Acceptance Corrected Data vs MC
$\Delta \eta$ for Different Gap Fractions}

\section{All $x_{\gamma}{ }^{\text {OBS }}$ ZEUS}
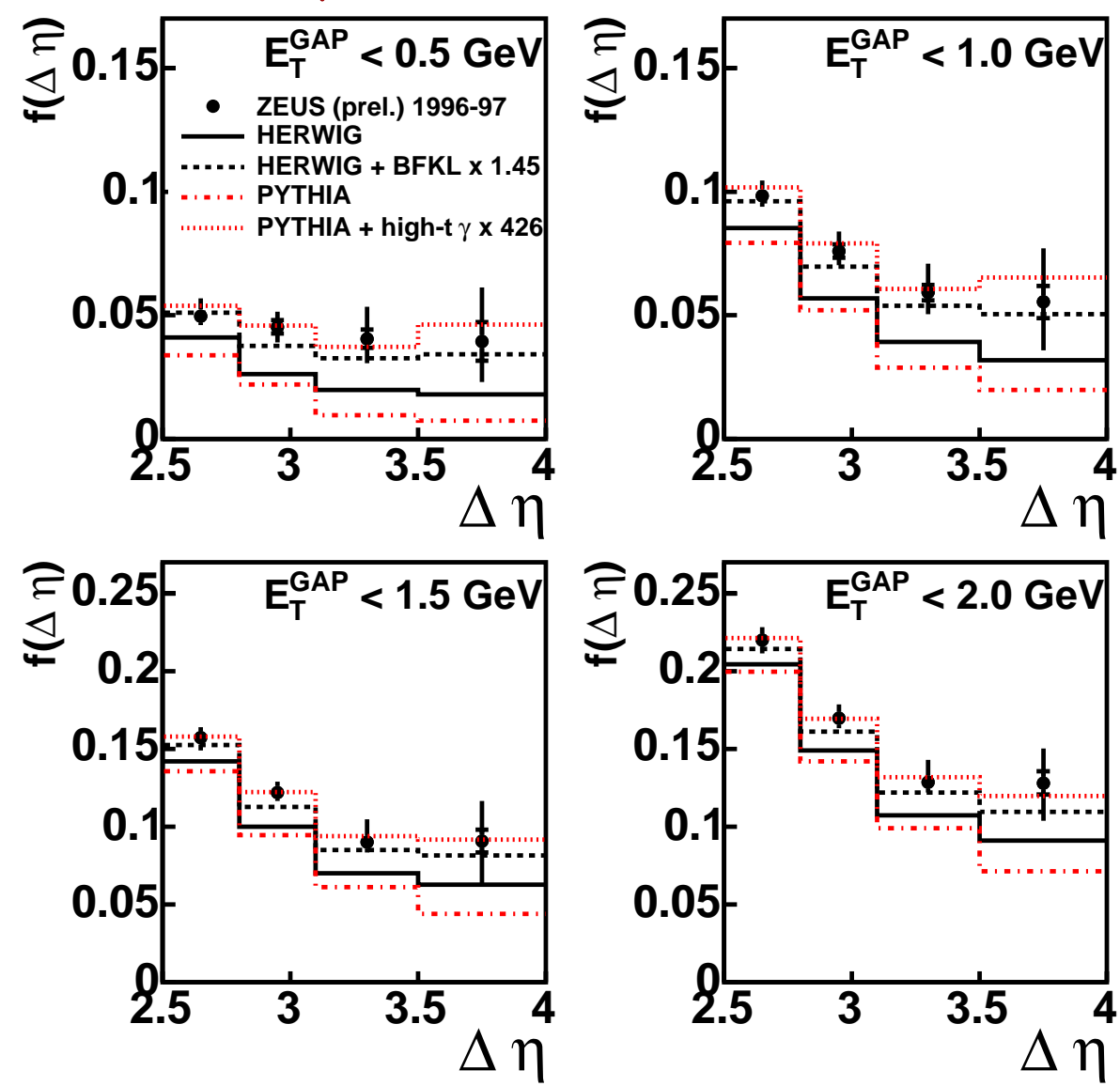

E0.25 $\quad \mathrm{E}_{\mathrm{T}}^{\mathrm{GAP}}<2.0 \mathrm{GeV}$ $\unlhd 0.2=$ $x_{y}{ }^{\mathrm{OBS}}<0.75$ ZEUS Resolved

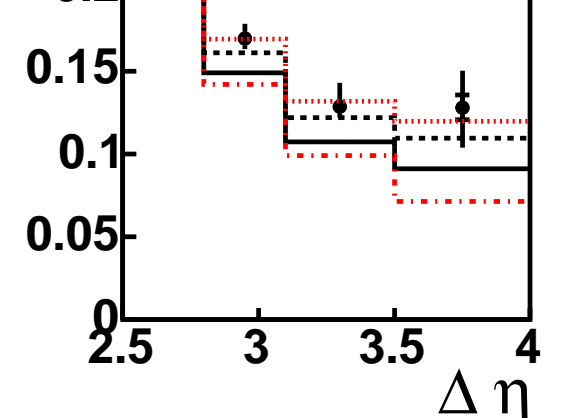

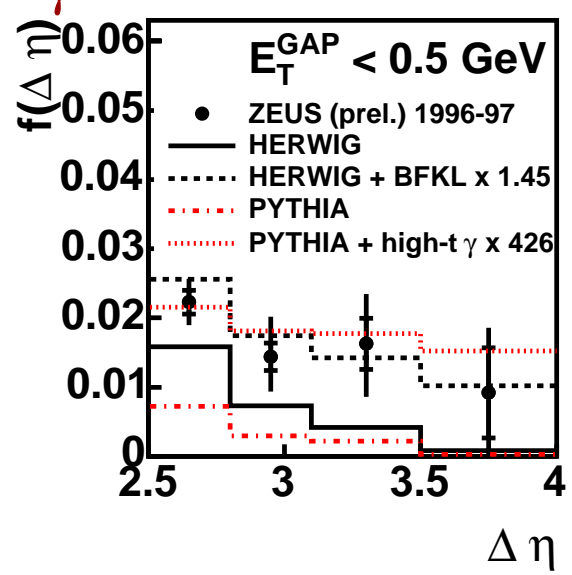

巳0.06 $\mathrm{E}_{\mathrm{T}}^{\mathrm{GAP}}<1.0 \mathrm{GeV}$ $\underset{\Downarrow}{\longleftarrow} 0.05$ 0.04 t

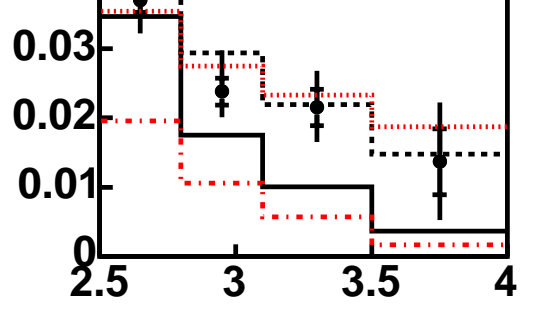

$\Delta \eta$
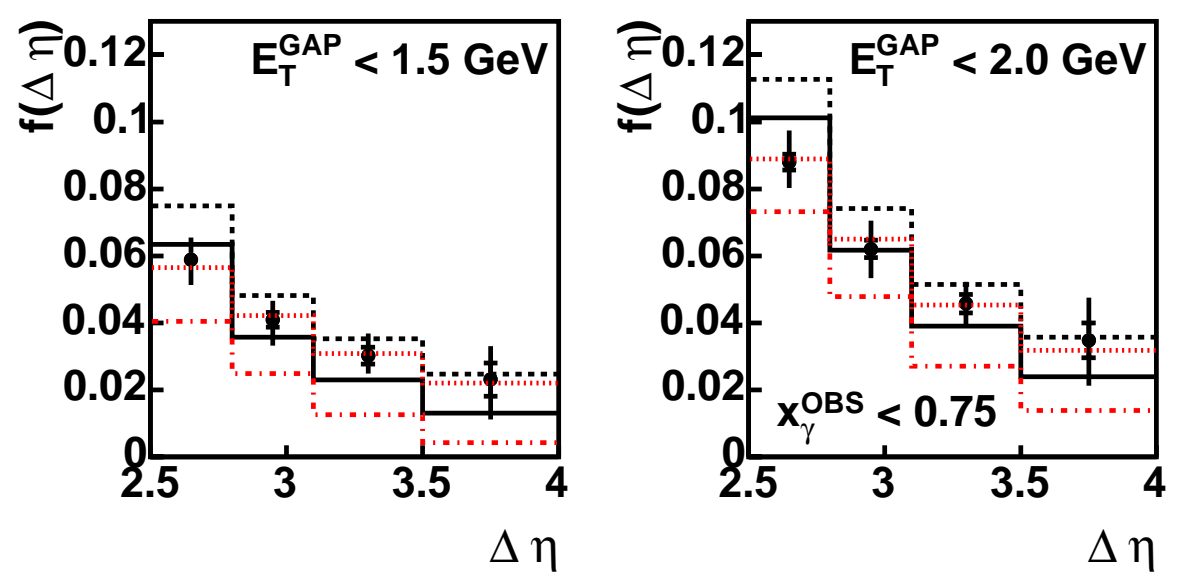

- MC with CS describes data for entire $x_{\gamma}{ }^{\text {OBS }}$ region

- CS contribution in resolved region is $1-2 \%$ 
ZEUS

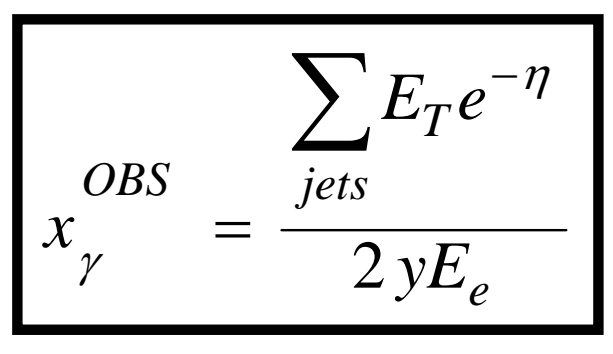

Resolved region: $\mathbf{x}_{\gamma}^{\text {OBS }}<0.75$

$$
\text { 哭 }
$$
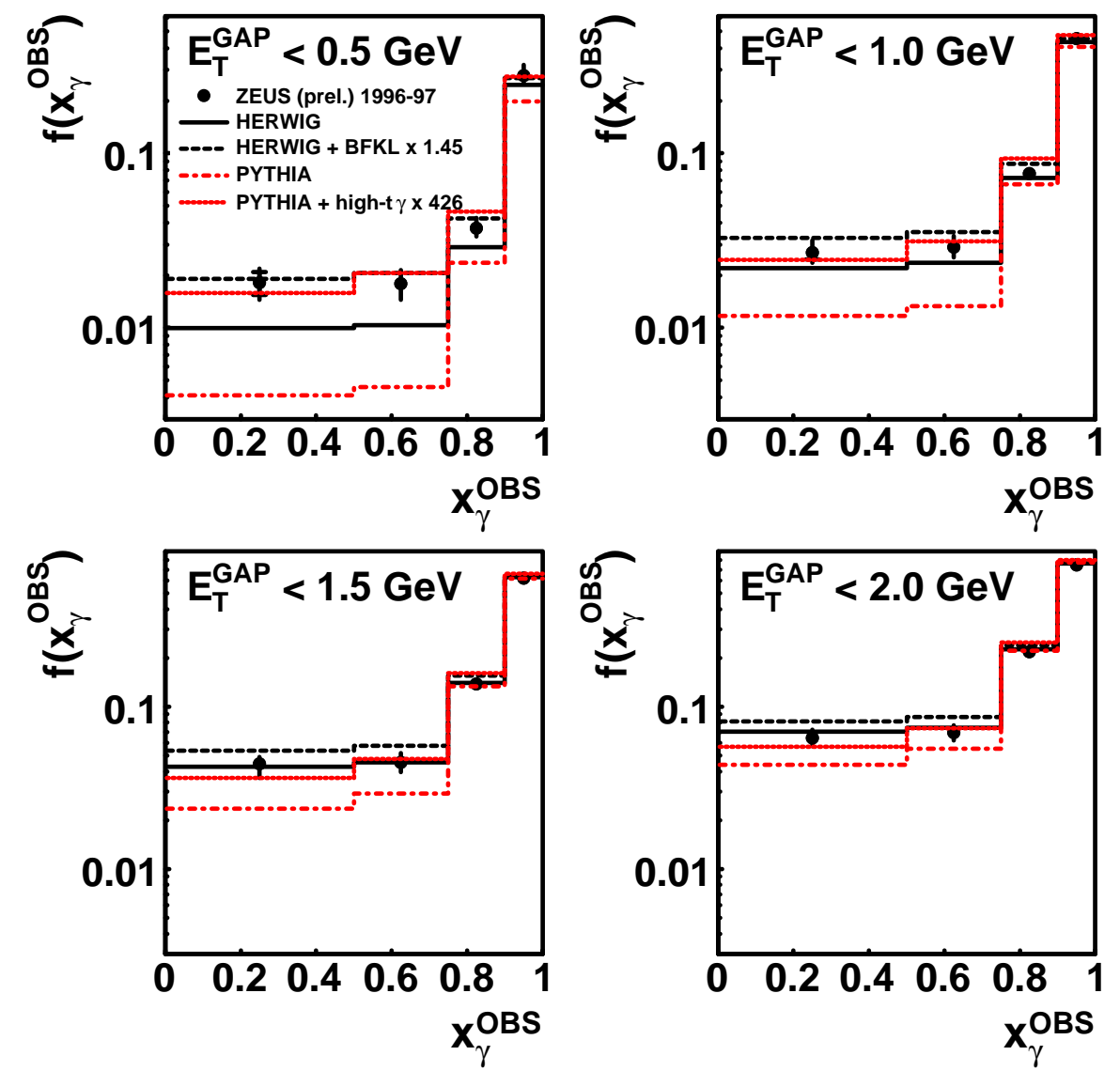

- MC with CS describes the Data

- HERWIG agreement remains better than PYTHIA agreement

- PYTHIA agreement in resolved region improved compared to $\Delta \eta$ 


\section{$\Delta \eta$ Gap Fractions Resummed Calculation}

- Resummed Calculation

- Seymour \& Appleby

- Only calculation available

- Large Errors

- Shape of data described

- $\mathrm{E}_{\mathrm{T}}^{\mathrm{Cut}}=0.5$

- Data above prediction

- All other $\mathrm{E}_{\mathrm{T}}$ cut values

- Data below predictions

- Disagreement increases as $\mathrm{E}_{\mathrm{T}}{ }_{\mathrm{Cut}}$ increases

\section{ZEUS}
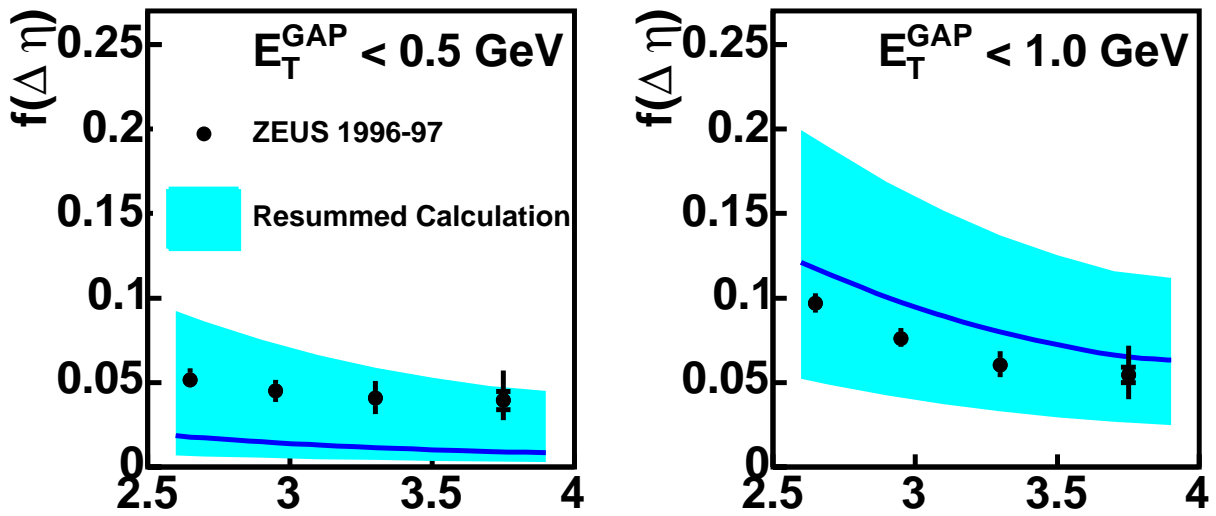

$\Delta \eta$

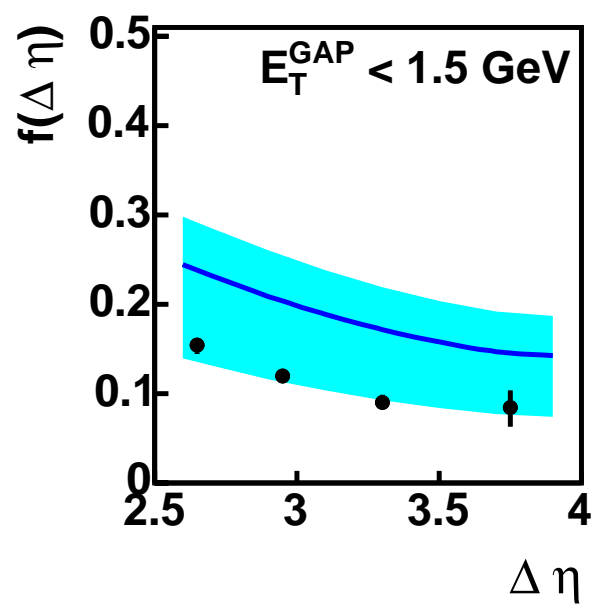

$\Delta \eta$

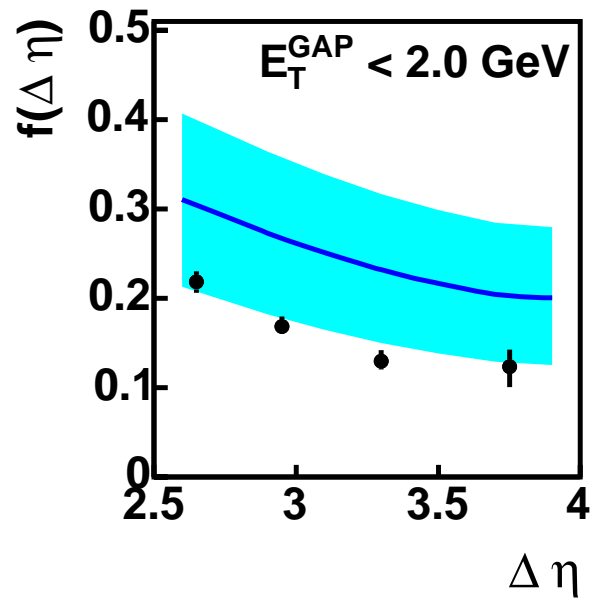




\section{ZÉus Rapidity Gap Between Jets
Summary}

\section{- Conclusions}

- Data demonstrate evidence of $\sim 3 \%$ Color-Singlet contribution estimated at the cross section level for entire phase space

- Corresponds to $\sim 1-2 \%$ Color-Singlet in resolved region

- Data consistent with published ZEUS and H1 results

- PYTHIA and HERWIG describe data well after the Color-Singlet contribution is added

- In Progress

- Examine W dependence

- Explore comparisons with Tevatron 